\title{
Quantitative Evaluation of the Polarization Ch
of Coronary Arteries Atherosclerotic Plaques at Different Development Stages
}

DOI: 10.17691/stm2015.7.4.05

Received October 13, 2015

E.V. Gubarkova, Junior Researcher, Laboratory for the Study of Optical Structure of Biotissue, Institute

of Biomedical Technologies";

E.B. Kiseleva, PhD, Junior Researcher, Laboratory for the Study of Optical Structure of Biotussie, Institute of Biomedical Technologies";

M.Yu. Kirillin, PhD, Senior Researcher, Laboratory of Biophotonics²;

L.B. Timofeeva, PhD, Researcher, Morphology Unit, Central Scientific Research Laboratory;

Assistant, Department of Histology with Cytology and Embryology';

S.S. Kuznetsov, MD, DSc, Head of Pathological Anatomy Department';

F.I. Feldchtein, PhD, Consultant for Biophotonics; Leading Researcher, Institute of Biomedical Technologies';

N.D. Gladkova, MD, DSc, Professor, Vice Director for Science, Institute of Biomedical Technologies ${ }^{1}$

${ }^{1}$ Nizhny Novgorod State Medical Academy, 10/1 Minin and Pozharsky Square, Nizhny Novgorod, 603005,

Russian Federation;

${ }^{2}$ Institute of Applied Physics, Russian Academy of Sciences, 46 Ulianova St., Nizhny Novgorod, 603950,

Russian Federation;

${ }^{3}$ Dental Photonics, Inc., 1600 Boston-Providence Highway, 02081, Walpole, USA

The aim of the investigation was to develop an approach to quantitative evaluation of polarization properties (birefringence and crossscattering) basing on cross-polarization OCT images (CP OCT) in order to characterize the development stages of atherosclerotic plaques and to reveal unstable ones.

Materials and Methods. We report on quantitative analysis of CP OCT images of the seven development stages of atherosclerotic plaques ex vivo. Integral depolarization factor (IDF) and effective birefringence coefficient $(\Delta n)$ were proposed as parameters for quantitative characterization of the CP OCT images.

Results. Calculation of the IDF and $\Delta n$ in the local region of interest (intima/fibrous cap) showed a statistically relevant difference between stable (stage IV) and unstable (stage Va) plaques $\left(0.46 \pm 0.21\right.$ against $0.09 \pm 0.04$ for IDF and $(0.47 \pm 0.10) \cdot 10^{-3}$ against $(0.25 \pm 0.07) \cdot 10^{-3}$ for $\Delta n$; $\mathrm{p}<0.05)$. It was found that $\Delta n$ value in the range $(0.22-0.29) \cdot 10^{-3}$ (within the limits of two standard deviations) indicates the presence of only a small amount of highly organized collagen in the fibrous cap of an unstable plaque which can indicate its tendency to rupture. We believe that these changes are connected with the prevalence of disorganized fibers during the inflammatory process in the fibrous cap of an unstable plaque, and to the presence of clusters of foam cells and inflammatory cells between them.

Conclusion. The proposed approach to the quantitative evaluation of CP OCT images (calculation of IDF and building $\Delta n$ maps) allows to assess both cross-scattering and birefringence of atherosclerotic plaques at various development stages and more reliably reveal their vulnerability.

Key words: cross-polarization optical coherence tomography; СP OCT; atherosclerotic plaque; quantitative image processing; crossscattering; effective coefficient of birefringence.

Advances in technologies for in vivo diagnosis and monitoring of atherosclerosis development based on evaluation of microstructural changes in vessel walls has been the main direction of development of intravascular visualization techniques. However, very few medical visualization techniques combine high spatial resolution with ability for intravascular application and simultaneous retrieval of key tissue characteristics.

Optical coherence tomography (OCT) technique meets the above criteria, as it provides two- and three- dimension structural images of a tissue at depths of up to $1-2 \mathrm{~mm}$ with the spatial resolution down to a few micrometers basing on real-time detection of backscattering.

Visual evaluation of OCT images of atherosclerotic plaques was done by many researchers $[1,2]$. Identification of the layers within the walls of a healthy vessel and the main components of a plaque, a fibrous cap, a lipid core, and calcium crystals, has been performed [3-6]. However, these studies have shown

For contacts: Gubarkova Ekaterina Vladimirovna, e-mail: kgybarkova@mail.ru 
that the contrast between the fibrous cap and the lipid core of the plaque in traditional OCT images is often insufficient for the visualization of these structures. As a result, recent studies were aimed at identification of typical features connected with the vulnerability of these plaques (the risk of instability), such as clusters of foam cells and inflammatory cells in the fibrous cap of the plaque, using intravascular OCT [7-10].

OCT modifications that use polarized light as the probing radiation allow to observe not only the scattering by plaque components, but also to identify structures that exhibit birefringence or cross-scattering, and can be of great help in solution of these problems. Polarization-sensitive OCT (PS OCT) is based on the mapping and analysis of a phase delay [11-13] whose rate of change with depth characterize the degree of structural organization and orientation of collagen fibers at the tissue level [14]. In paper [15] visual evaluation of the degree of birefringence change in samples of coronary arteries in PS OCT images was compared to the properties of collagen in preparations stained with picrosirius red. Nadkarni et al. [11, 16] analyzed PS OCT images of athersclerotic plaques with high and low collagen content and different density of smooth muscle cells. They revealed a high positive correlation between birefringence measured in PS OCT images and the accumulation of collagen in a plaque $(r=0.67 ; p<0.001)$; as well as the correlation between the presence of high content of thick collagen fibers $(r=0.76 ; p<0.001)$ and the density of the smooth muscle cells $(r=0.74 ; p<0.01)$.

At the same time, Kuo et al. [17, 18] developed an algorithm for complex qualitative analysis of PS OCT images that allows evaluation of both scattering and birefringence properties of vascular walls for the main stages of atherosclerosis. It was shown that the birefringence coefficient $(\Delta n)$ of fibrous and fibrocalcified plaques, the dominant extracellular component of which is thick collagen bundles, was significantly higher than that of a normal vascular wall where only a small amount of thin collagen fibers presents $\left(9.4 \cdot 10^{-4}\right.$ against 5.3.10-4; $p<0.05)$. However, this approach does not allow for differentiation of the stages of plaque development basing on presence of lipids/a lipid core that determines a serious risk of plaque rupture (an "unstable" plaque). One can suppose that the presence of lipids in the fibrous cap causes multiple scattering of linearly polarized radiation (which is used as the probing radiation in PS OCT systems) due to increased heterogeneity of medium. Furthermore, as disorganized fibers of the capsule lose their birefringence, the use of PS OCT in this situation becomes ineffective.

It should be noted that in the papers by Nadkarni et al. $[11,16]$ and Kuo et al. $[17,18]$ the region of interest for quantitative evaluation in the PS OCT images was determined manually basing on histological data and analysis of the signal behavior. The results of PS OCT images processing can be considered as an attempt to characterize, quantitatively, the properties of an atherosclerotic plaque at different stages of its formation, however, not as criteria for independent PS OCT diagnosis.

We took this approach as a starting point and applied it to cross-polarization (CP) OCT images. In the employed CP OCT system circularly polarized light is used for object inspection and the signals are registered in two channels corresponding to co-scattering and cross-scattering, respectively [19]. This approach provides certain advantages for quantitative evaluation of changes in polarization properties of intima or fibrous cap during different stages of formation of atherosclerotic plaque. For example, CP OCT can reliably and reproducibly register such polarization effects as local cross-scattering and birefringence which is important in developing quantitative criteria for assessing the progress of atherosclerosis.

Earlier [20, 21] CP OCT systems were demonstrated to allow both better revealing structures with optical anisotropy and monitoring their changes. Furthermore, in our previous work [22] we showed that the CP OCT method can increase both the reliability and accuracy of visual evaluation of the atherosclerotic plaques microstructure due to sensitivity to birefringence and cross-scattering phenomena in biotissues.

The papers $[23,24]$ have presented the results of quantitative processing of CP OCT images of mucous membranes of human internal organs by calculating the integral depolarization factor (IDF). IDF was employed for in vivo identification of pathophysiological processes (inflammation, fibrosis and neoplasia) connected mainly with changes in spatial and structural organization of collagen fibers. However, pathological processes studied in these papers, unlike formation of atherosclerotic plaques, involved considerable regions of organ in indepth direction, so cross-scattering (depolarization of radiation) dominated among the polarization effects, and IDF calculated automatically for all the CP OCT images provided high level of diagnostic accuracy.

Our team was the first to develop algorithms for quantitative processing of $\mathrm{CP}$ OCT images to enable characterization of atherosclerotic plaques development stages in coronary arteries, according to their birefringence and cross-scattering properties. These polarization effects cannot be completely separated from each other, however, their versatile quantitative evaluation will increase the reliability and accuracy of microstructure image interpretation and help in identification of the plaques composition at different atherosclerosis development stages.

The aim of the investigation was to develop an approach for quantitative evaluation of tissue polarization properties (birefringence and cross-scattering) from cross-polarization OCT images in order to characterize the development stages of atherosclerotic plaques and to reveal their instability. 
Materials and Methods. The objects under study were 60 post mortem samples of coronary arteries of patients (two males and six females, in the age range 65-90) died from cardiovascular disease. All the samples were obtained during autopsy according to the scientific research protocol approved by the Ethical Committee of the Nizhny Novgorod State Medical Academy (Protocol No.7 from 26 August 2014). The arterial vessels obtained not later than $24 \mathrm{~h}$ after death were cut into fragments with length of $1-2 \mathrm{~cm}$ and delivered to the laboratory in gauze moistened with phosphate buffer at $7^{\circ} \mathrm{C}$.

The spectral domain CP OCT device designed and developed at the Institute of Applied Physics of the Russian Academy of Sciences (Nizhny Novgorod) [2527] was employed in the study. The light source was a superluminescent diode with a central wavelength of $1,310 \mathrm{~nm}$, a spectrum width of $100 \mathrm{~nm}$, radiation power of $20 \mathrm{~mW}$ resulting in axial resolution of $10 \mu \mathrm{m}$. The device simultaneously builds two conjugated images in co- (top image) and cross- (bottom image) polarization.

The CP OCT images were obtained from the intima side of fresh arterial samples (within $2 \mathrm{~h}$ after excision). The direction and the range of scanning were: $4 \mathrm{~mm}$ along the vessel and $1 \mathrm{~mm}$ in-depth, respectively. After obtaining CP OCT images of the sample, an area around the OCT probe (6 $\mathrm{mm}$ in diameter) was marked, then the sample was fixed (in 10\% formalin for $48 \mathrm{~h}$ ) and serial sections of the central area aligned with the plane of the CP OCT images were taken. These sections were stained with hematoxylin and eosin and imaged in transmitted light with Leica DM2500 DFC (Leica Microsystems, Germany) microscope equipped with a digital camera. All the samples of coronary arteries were divided by histoautopsists into groups according to the development stage of atherosclerosis: insignificant thickening of the intima (stage I), thickening of the intima, with the deposition of lipids (stages II/III), a stable plaque with a fibrous cap and a lipid core (stage IV), an unstable plaque with a thin fibrous cap and a lipid core (stage $\mathrm{Va}$ ), a fibro-calcified plaque (stage $\mathrm{Vb}$ ) and fibroatheroma with pronounced fibrosis (stage $\mathrm{Vc}$ ) [28-30].

A total of 108 pairs of CP OCT and microscope images were obtained and analyzed.

The region of interest $(\mathrm{ROI})$ for quantitative processing was chosen manually basing on histological data and quantitative characteristics of the CP OCT image. This $\mathrm{ROI}$ deliberately contained only the upper part of the atherosclerotic plaque, namely thickened intima up to the media for stages I-III, the fibrous cap with the lipid core for stage IV, the fibrous cap above the lipid core for stage $\mathrm{Va}$, the fibrous cap without calcium accumulations for stage $\mathrm{Vb}$, and the thickened fibrous cap for stage Vc. This manual segmentation of the image allowed for quantitative determination of the polarization properties of accurately specified structures of the atherosclerotic plaque.

Numerical processing of the CP OCT images involved calculation of the IDF and effective birefringence coefficient $(\Delta n)$ for selected ROI.

In this study we used previously developed [23, 31, 32] algorithm for calculation of IDF, earlier applied for evaluation of the state of the collagen fibers in a variety of tissues (bladder mucosa, mouth cavity, and aorta). The calculation of IDF is based on the ratio of the OCT signal levels in co- and cross-polarization averaged over ROI.

Similar to mapping the birefringence coefficient from PS OCT images [18, 33], corresponding maps can also be built from CP OCT images (although they have a different formation principle) for effective birefringence coefficient $(\Delta n)$ as in-depth derivative of the effective phase delay:

$$
\Delta n(x, z)=\frac{1}{k_{0}} \frac{\partial}{\partial z} \operatorname{arctg}\left(\frac{10^{I x y}(x, z) / 10}{10^{I x x}(x, z) / 10}\right),
$$

where $I_{x x}(x, z)$ and $I_{x y}(x, z)$ represent the levels of OCT signals in the co- and cross-polarizations, respectively, expressed in decibels, and $\mathrm{k}_{0}$ is a wave vector.

We treat the phase delay and the resulting birefringence coefficient as effective values, because both processes, birefringence and cross-scattering, take part in formation of the CP OCT signal and are hard to be separated.

Due to speckle structure of OCT signal pixel-bypixel calculation of the OCT signal ration may result in high noise in calculated $\Delta n$ value. To overcome this problem, prior to calculation of $\Delta n$ spatial averaging of OCT signal both in the co- and cross-polarizations was performed (adjacent averaging, averaging over 10 pixels). Additionally, the signal values in crosspolarization not exceeding the level of the noise were set to zero, because they do not provide useful contribution to calculated $\Delta n$ map.

Statistical analysis of results was performed using Real Statistics for MS Excel and GraphPad Prism 6 software. The mean $(M)$ values of the measured parameters and the standard deviations of the mean $( \pm S D)$ were calculated. Significance of differences between groups was calculated with non-parametric Mann-Whitney test. In all cases the differences at the $p<0.05$ level of relevance were considered statistically significant. Confidence intervals were treated as $\mathrm{M} \pm 2 \mathrm{SD}$.

Results and Discussion. Employed high-speed spectral domain CP OCT setup detects OCT signal in two polarizations, parallel (co-) and orthogonal (cross-) to the initial one, and builds corresponding OCT images. The correlation between the levels of OCT signals in cross- and co-polarization in particular point depends on the local cross-scattering and on birefringence above the considered area.

Analysis of CP OCT images and effective birefringence coefficient maps of coronary arteries. The key feature of the initial development stage of an atherosclerotic plaque (stage I) is a small thickening of the intima due to the increased synthesis of type I 
collagen fibers (Figure 1 (a)). In CP OCT images obtained, all the layers (intima, media and adventitia) can be well differentiated both in co- and cross-polarizations (Figure 1 (c)). The thickened intima demonstrates a high level of backscattering, while the media, as a rule, exhibits a lower level. The adventitia is usually manifested by heterogeneous, but high level of backscattering. In the $\Delta n$ map in selected $\mathrm{ROI}$ (black rectangle in Figure $1(\mathrm{e})$ ) one can observe the change in polarization properties of a slightly thickened intima. One can see that higher $\Delta n$ values with more homogeneous distribution along the transverse coordinate prevail. Along with the histology results, this indicates the presence of organized and structured collagen fibers in the studied area.
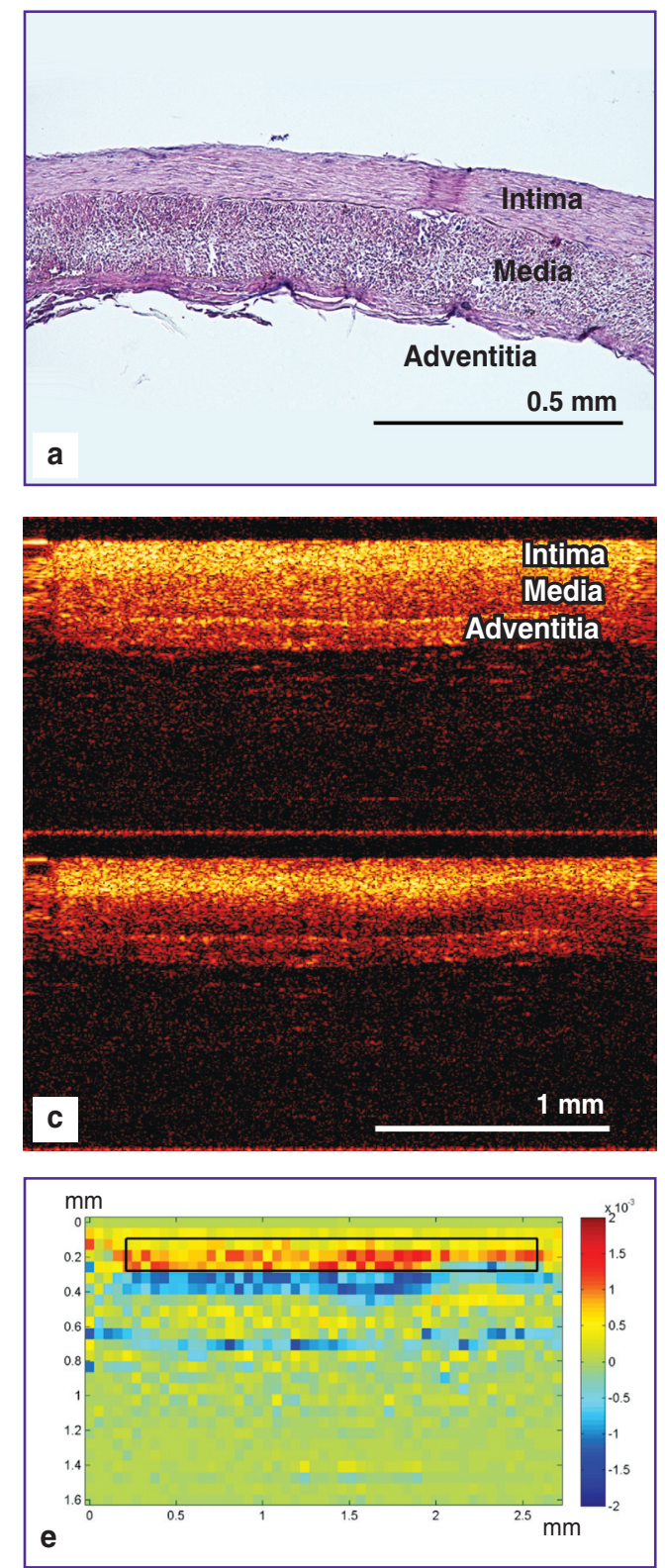

In the case when lipids are deposed on the vascular wall (stages II/III of plaque development), a slight disordering in organization of the collagen and elastic fibers occurs in the thickened intima manifested by their weak fibrinoid swelling and partial fragmentation (Figure 1 (b)). At the same time the cross-polarization channel shows a decrease in the level of backscattering from the surface (Figure 1 (d), arrow). Additionally, the $\Delta n$ map demonstrates a decrease of values and disturbance in homogeneity of the $\Delta n$ distribution within the area of the thickened intima (Figure 1 (f)) compared to stage I (Figure 1 (e)).

Stable and unstable atherosclerotic plaques (stages IV and $\mathrm{Va}$, respectively) can be similar in size and do not
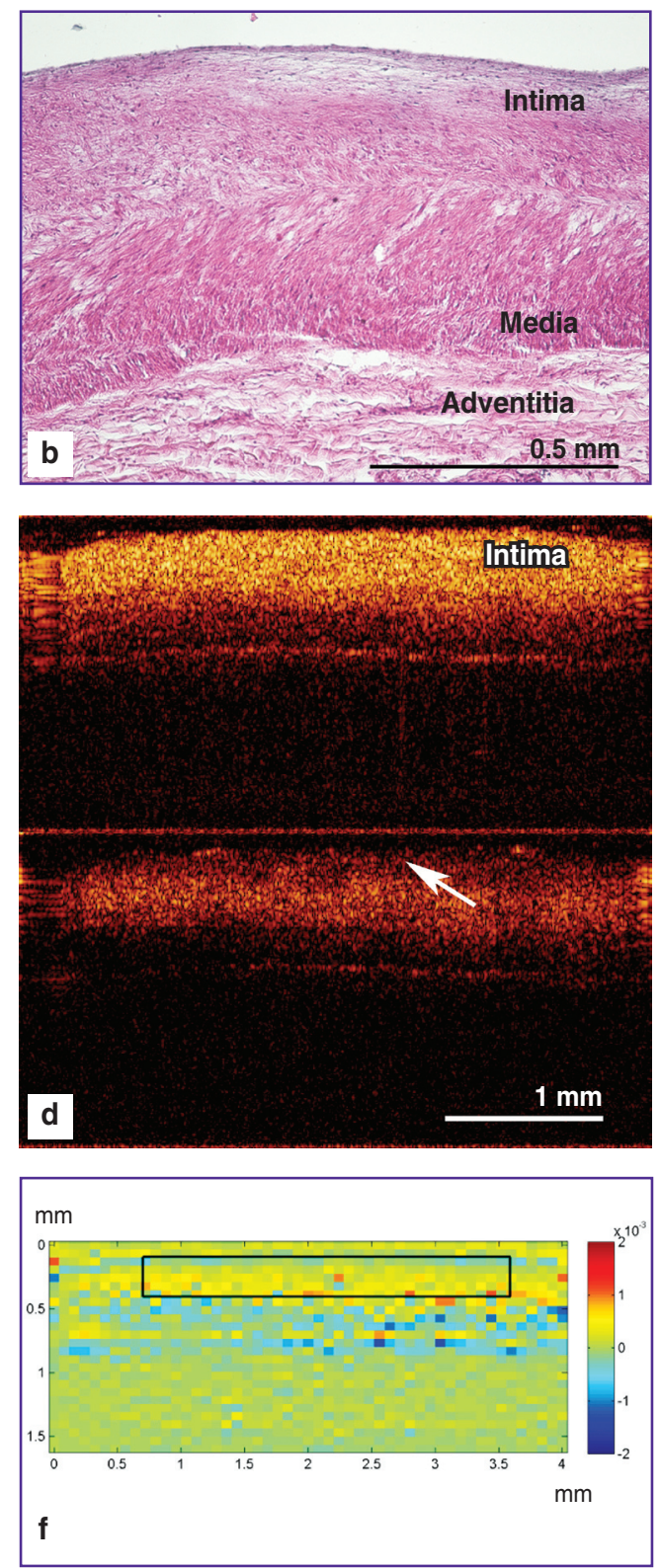

Figure 1. Histological and CP OCT images of the thickened intima of insignificantly changed coronary artery wall at plaque stage I (left column) and plaque stage II with the lipid deposition (right column); histological preparations stained with hematoxylin and eosin (a), (b); CP OCT images in co-polarization (top) and cross-polarization (bottom) (c), (d); $\Delta n$ maps (e), (f) 
lead to visible narrowing in the vessel lumen. They are therefore difficult to differentiate by means of common intravascular imaging techniques [34]. However, internal structure of both fibrous cap and lipid core for these stages is completely different.

Fibroatheroma (stage IV) as a rule is characterized by the presence of a small lipid core covered with a fibrous cap (Figure 2 (a)). The fibrous cap mainly contains densely packed, organized collagen and elastic fibers in a fibrinoid swelling phase ensuring stability of the plaque. In cross-polarization CP OCT image a dark stripe caused by birefringence is visible (Figure 2 (c)), lower image, arrow). The $\Delta n$ map in the area of the fibrous components of the fibrous cap Figure 2 (e)) is characterized by high values, while the area of lipid clusters corresponds to lower ones.

Morphologically the plaque stage Va, unlike stage IV, has an unstable fibrous cap that contains fibers of collagen and elastin, disorganized in course of inflammation and located above the lipid core, and clusters of foam and inflammatory cells (Figure 2 (b)). In co-polarization CP OCT image, the upper layer of the fibrous cap appears as an area with a highly heterogeneous OCT signal rapidly degrading with depth (Figure 2 (d), top image). In crosspolarization the corresponding area demonstrates a heterogeneous OCT signal featuring conglomerates with high signal level which provide jumps in cross-scattering (Figure $2(\mathrm{~d})$, bottom image, arrow). This signal indicates
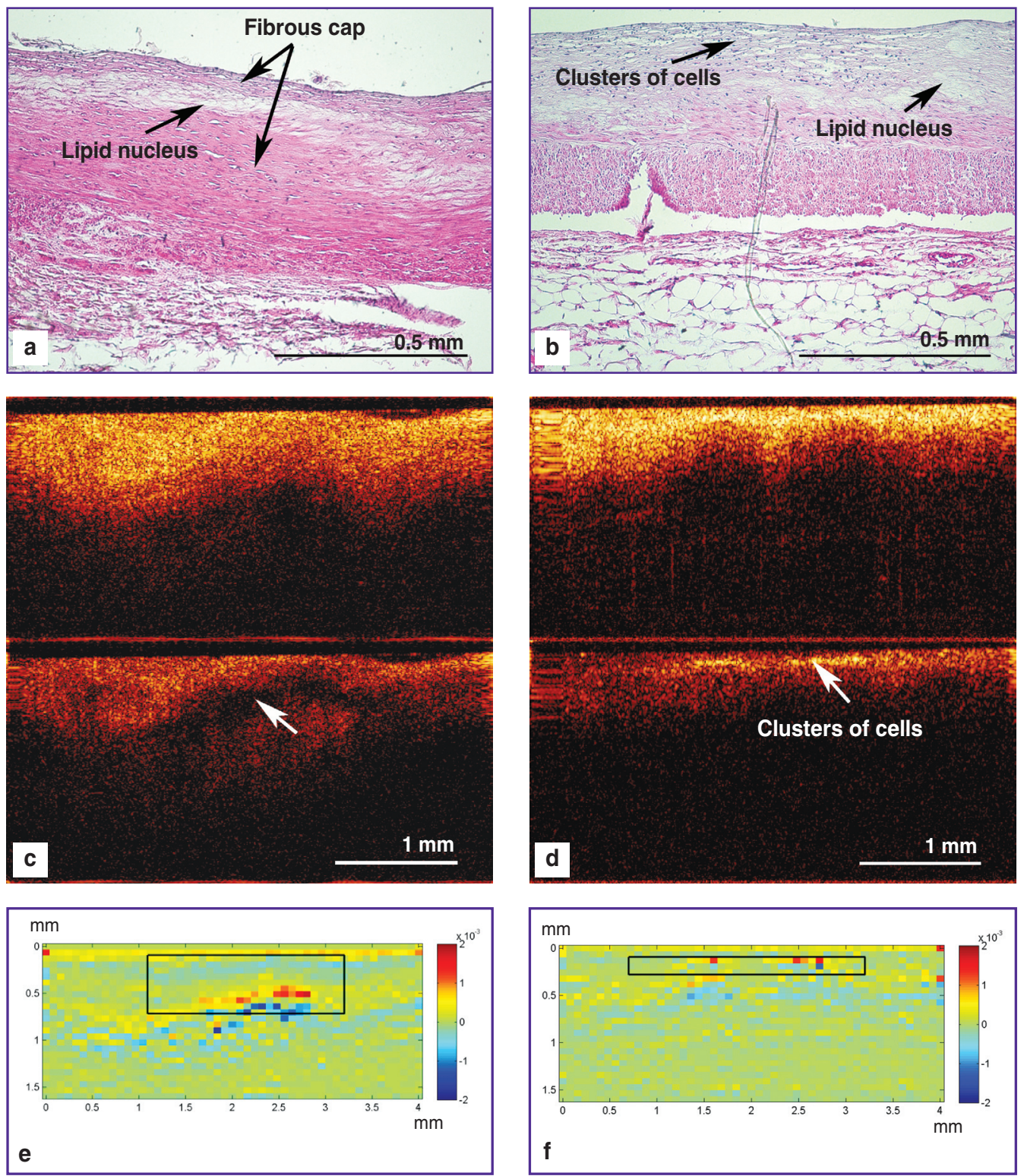

Figure 2. Histological and CP OCT images of stable (stage IV, left column) and unstable (stage Va, right column) atherosclerotic plaques; histological preparations stained with hematoxylin and eosin (a), (b); CP OCT images in co-polarization (top) and crosspolarization (bottom) (c), (d); $\Delta n$ maps (e), (f) 
the presence of clusters of inflammatory and foam cells in the area of destroyed fibers of the fibrous cap. When analyzing the image, this effect can point to instability of the plaque. Accordingly, in the $\Delta n$ map, such jumps in the cross-scattering are manifested by areas with high $\Delta n$ values against a background of low values originating from the presence of disorganized fibers in the thin fibrous cap (Figure $2(f)$, black rectangle).

In CP OCT images, the plaque with calcification (stage $\mathrm{Vb}$ ) in co-polarization is visualized as an area with high signal level and a sharp boundary in the region of accumulation of the calcium salts in the lipid core (Figure 3 (c), upper image). This matches traditional OCT images obtained by other researchers, for example [3]. However, CP OCT image provides more information about the state of the fibrous cap in this type of plaque. In both co- and cross-polarization, a high level of backscattering can be observed (Figure 3 (c)) indicating the presence of organized collagen fibers in the plaque (unlike the unstable plaque). In this case the fibers are at the stage of fibrinoid swelling preserving, however, their coaxial orientation along the vessel lumen and, therefore, the stability of the plaque fibrous cap (Figure $3(a)$ ). In the $\Delta n$ map, mainly higher values prevail, however, with significant variations in transversal direction (Figure 3 (e), black rectangle).

In CP OCT image of fibroatheroma with pronounced fibrosis (stage Vc) organized fibers of collagen located in the thickened fibrous cap (Figure 3 (b)) reveal pronounced birefringence (Figure $3(\mathrm{~d})$ ). In the $\Delta n$ map
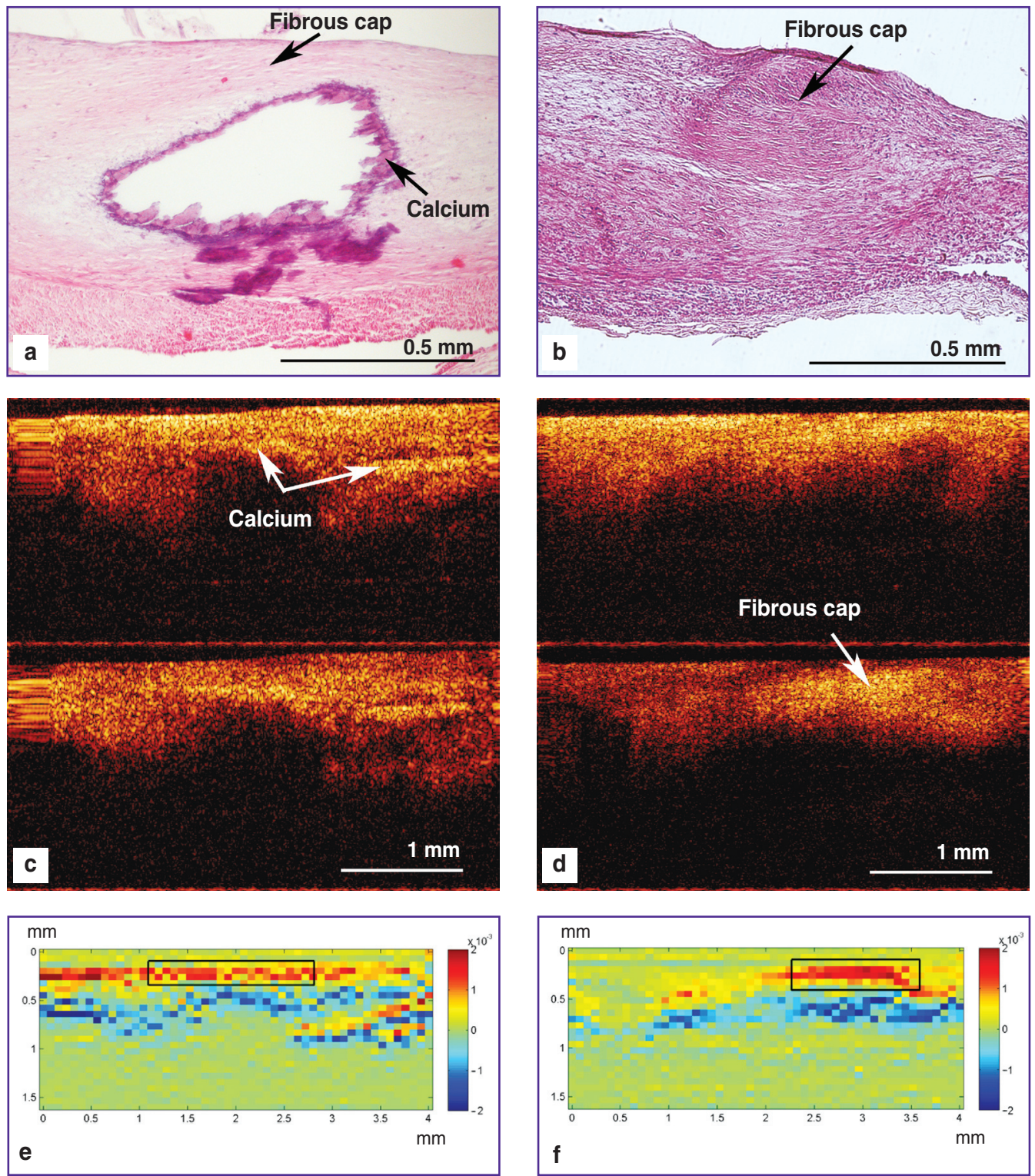

Figure 3. Histological and CP OCT images of the atherosclerotic plaque with calcification (stage $\mathrm{Vb}$, left column) and with pronounced fibrosis (stage Vc, right column); histological preparations stained with hematoxylin and eosin (a), (b); CP OCT images in co-polarization (top) and cross-polarization (bottom) (c), (d); $\Delta n$ maps (e), (f) 
the values are mostly high, however, on the contrary to stage $\mathrm{Vb}$, are more homogeneous in the transversal direction (Figure $3(\mathrm{f})$ ).

Concluding on CP OCT efficiency in imaging different stages of atherosclerotic plaques of human coronary arteries it should be noted that the analysis of maps of the effective birefringence coefficient $\Delta n$ in the region of interest allows for more accurate interpretation of the images in co- and cross-polarizations. At the initial stages of atherosclerotic plaque development (thickened intima) the $\Delta n$ maps show high level of cross-scattering and homogenous distribution of $\Delta n$ in transversal direction (Figure 1 (e)). In the $\Delta n$ maps for fibrous and fibrocalcified plaques, $\Delta n$ also demonstrates high values in the area of organized fibers, however, with less homogeneous distribution in transversal direction (Figure $3(\mathrm{e}),(\mathrm{f})$ )) Furthermore, the $\Delta n$ maps for plaque development stages II/III, IV and Va show primarily heterogeneous distribution of $\Delta n$ values along the transversal direction (Figures 1 (f) and 2 (e), (f)). These changes are associated with accumulation of lipids in the intima, at stages II/III and IV, and prevalence of disorganized fibers in the fibrous cap of the unstable plaque at stage $\mathrm{Va}$, which leads to sharp jumps of the signal in cross polarization.

Numerical processing of CP OCT images. It should be noted that when studying atherosclerosis of human coronary arteries with normal wall thickness of $\sim 1 \mathrm{~mm}$, the interest is focused in the local area (intima or fibrous cap) with thickness down to $65 \mu \mathrm{m}$ [35]. In the most cases, this requires segmentation of the $\mathrm{CP}$
OCT image with further calculation of characteristics of selected ROI.

In this study we performed quantitative evaluation of polarization characteristics (cross-scattering and birefringence) of atherosclerotic plaques only for ROI (the thickened intima or the fibrous cap) selected based on the histology data.

1. The results of the IDF calculation (Table 1) demonstrate statistically significant difference $(p<0.05)$ between initial (I) and "final" stages of plaque development, unstable plaque $(\mathrm{Va})$ and firboatheroma with pronounced fibrosis (stage Vc) (Figure 4). With IDF one can therefore differentiate clinically significant states of coronary arteries plaques, stages IV and Va $(p<0.05)$, both having fibrous caps and a lipid core, but differing in the state of the fibrous cap.

Thus, IDF allows characterizing local tissue birefringence and ability for cross-scattering, while low IDF values in the ROI indicate disordering of the collagen and elastic fibers in the plaque fibrous cap.

2. The effective birefringence coefficient $(\Delta n)$ characterizes the local difference in refractive indices of the ordinary and extraordinary waves within particular structural components of an atherosclerotic plaque.

Within the ROI in $\Delta n$ map we calculated the mean value of $\Delta n$ which characterizes local birefringence and cross-scattering thus allowing to reliably determine areas with disorders in collagen and elastic fibers of stable or unstable plaques. The results of the calculation of the effective $\Delta n$ (Table 2, Figure 5) in the ROI of CP OCT

Table 1

Calculated values of integral depolarization factor (IDF) in CP OCT images and confidence intervals (CI) for different development stages of an atherosclerotic plaque

\begin{tabular}{|c|c|c|c|c|c|c|}
\hline IDF & Stage I (n=11) & Stage II/III (n=17) & Stage IV ( $n=13)$ & Stage Va $(n=19)$ & Stage $V b(n=27)$ & Stage Vc $(n=21)$ \\
\hline $\mathrm{M} \pm \mathrm{SD}$ & $0.51 \pm 0.04$ & $0.14 \pm 0.05$ & $0.46 \pm 0.21$ & $0.09 \pm 0.04$ & $0.41 \pm 0.19$ & $1.06 \pm 0.46$ \\
\hline $95 \% \mathrm{Cl}$ & $0.47-0.55$ & $0.11-0.16$ & $0.29-0.62$ & $0.07-0.12$ & $0.31-0.51$ & $0.83-1.29$ \\
\hline
\end{tabular}

Figure 4. Integral depolarization factor for all development stages of an atherosclerotic plaque. * indicates statistically significant differences between plaque development stages I, Va and Vc, $\mathrm{p}<0.05$; ** indicates difference between stable (IV) and unstable (Va) plaque stages, $\mathrm{p}<0.05$ (Mann-Whitney test)

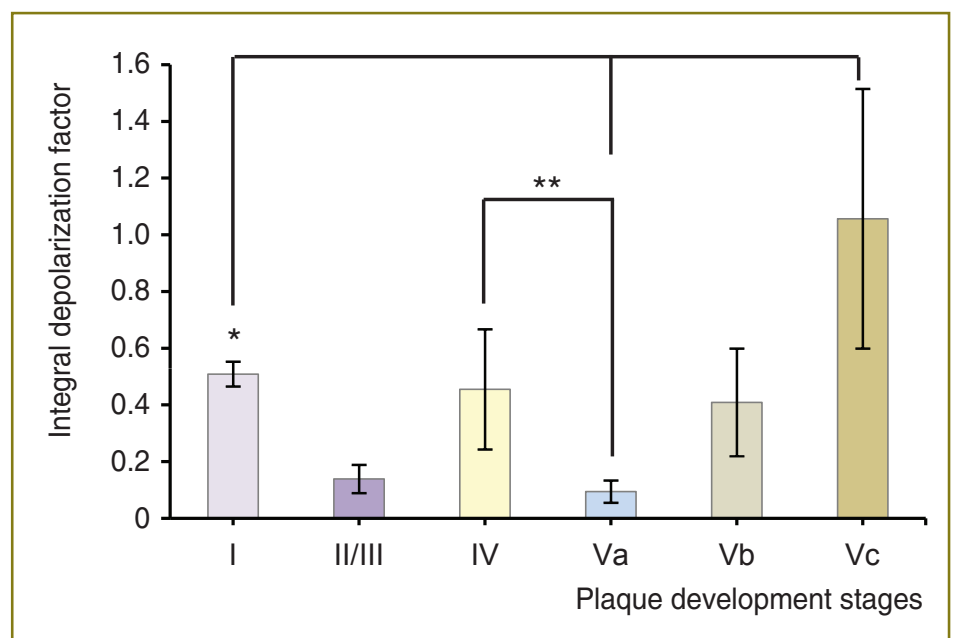


Table 2

Calculated values of effective birefringence coefficients $(\Delta n)$ in CP OCT images and confidence intervals $(\mathrm{Cl})$ for different development stages of an atherosclerotic plaque

\begin{tabular}{ccccccc}
\hline \multicolumn{1}{c}{$\Delta \boldsymbol{n}$} & $\begin{array}{c}\text { Stage I } \\
(\mathbf{n = 1 7})\end{array}$ & $\begin{array}{c}\text { Stage II/III } \\
(\mathbf{n}=\mathbf{1 3})\end{array}$ & $\begin{array}{c}\text { Stage IV } \\
(\mathbf{n = 1 9 )}\end{array}$ & $\begin{array}{c}\text { Stage Va } \\
(\mathbf{n = 2 7 )}\end{array}$ & $\begin{array}{c}\text { Stage Vb } \\
(\mathbf{n}=\mathbf{2 1})\end{array}$ & $\begin{array}{c}\text { Stage Vc } \\
(\mathbf{n}=\mathbf{2 1})\end{array}$ \\
\hline$(\mathrm{M} \pm \mathrm{SD}) \cdot 10^{-3}$ & $0.86 \pm 0.10$ & $0.29 \pm 0.07$ & $0.47 \pm 0.10$ & $0.25 \pm 0.07$ & $0.49 \pm 0.09$ & $0.58 \pm 0.12$ \\
\hline$(95 \% \mathrm{Cl}) \cdot 10^{-3}$ & $0.77-0.95$ & $0.25-0.32$ & $0.40-0.54$ & $0.22-0.29$ & $0.45-0.54$ & $0.52-0.64$ \\
\hline
\end{tabular}

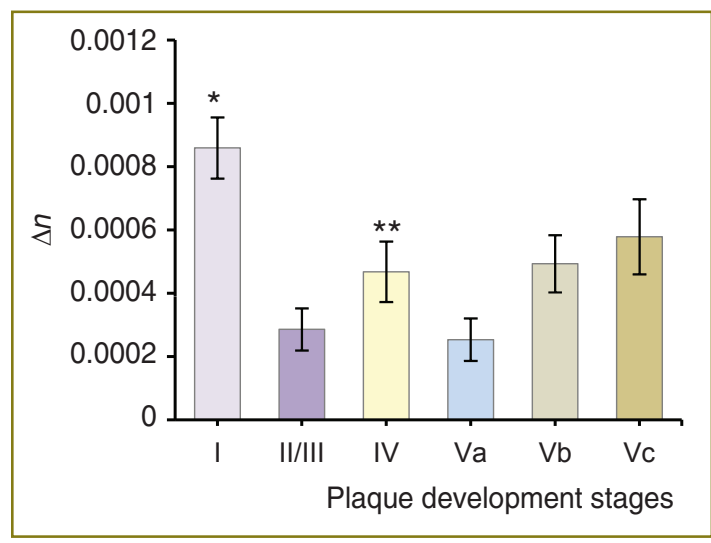

Figure 5. Effective birefringence coefficient $\Delta n$ for all development stages of an atherosclerotic plaque. * indicates statistically significant difference between stages I and all subsequent development stages of a plaque, $p<0.05$; ** indicates difference between stable (IV) and unstable (Va) plaque stages, $\mathrm{p}<0.05$ (Mann-Whitney test)

images at different stages of atherosclerotic plaque development revealed following features:

high mean values of $\Delta n$ indicate the presence of a large number of ordered anisotropic structures (collagen and elastic fibers) distributed homogeneously in the thickened intima/fibrous cap, at stages I, IV, Vb and Vc;

low mean values of $\Delta n$ indicate insignificant amounts of weakly ordered anisotropic structures (collagen and elastic fibers), distributed heterogeneously in the thickened intima at the initial stage of lipids deposition (II/III), and in the thin (unstable) fibrous cap at stage Va;

the presence of clusters of inflammatory cells at stage Va causes high local values in the $\Delta n$ map which can serve as an indicator for plaque instability.

The study revealed that the mean $\Delta n$ of organized and ordered collagen and elastic fibers in the insignificantly thickened intima at the initial stage of plaque development (stage I) is statistically $(p<0.05)$ higher than that of the disorganized fibers of subsequent stages of plaque development. At the same time, high values of the mean $\Delta n$ at stages I, IV, $\mathrm{Vb}$ and $\mathrm{Vc}$ indicate prevalence of organized collagen and elastic fibers in the intima or in the fibrous caps of plaques, while low values of the mean $\Delta n$ at stages $I / / I I$ and Va indicate the prevalence of disorganized, partially destroyed collagen and elastic fibers in the intima or in the fibrous cap.
An important result of the study is the revealed statistically significant differences in the values of employed parameters for unstable (stage $\mathrm{Va}$ ) and stable (stage IV) plaques: IDF of 0.09 against $0.46(p<0.05)$; and $\Delta n$ of $0.25 \cdot 10^{-3}$ against $0.47 \cdot 10^{-3}(\mathrm{p}<0.05)$ (Figures 4 and 5) which proves the significant disorganization of the structures responsible for the polarization properties at stage Va. Additionally, it was revealed that $\Delta n$ values in the range $(0.22-0.29) \cdot 10^{-3}$ (within the limits of two standard deviations) indicate low amount of highly organized collagen in the fibrous cap of an unstable plaque, a situation which can indicate its tendency to rupture.

When calculating the IDF and the mean $\Delta n$ we used an approach involving the manual, subjective selection of the ROI in the $\Delta n$ maps. Therefore, the results of the CP OCT images processing are treated as a measure of polarization properties of atherosclerotic plaque at different development stages, rather than criteria for independent plaque diagnostics by CP OCT technique. Nevertheless, the results of this study are an important step towards numerical processing of the CP OCT images of coronary arteries atherosclerotic plaques, and can become the basis of enhanced algorithms for the CP OCT images automated processing (including automated segmentation) aiming for differential diagnostics.

Conclusion. Maps of effective birefringence coefficient derived from CP OCT images enable assessment of organization and orientation of anisotropic structures in atherosclerotic plaques when studying of the pathologic changes in walls of human coronary arteries.

Calculation of the depolarization integral factor (IDF) and the effective birefringence coefficient $(\Delta n)$ provide important information about the local functional state of the intima/fibrous cap of coronary artery plaques. These quantitative characteristics of cross-scattering and birefringence within anisotropic structures of coronary arteries helped in differentiation of the functional state of collagen and elastic fibers in the fibrous cap of unstable and stable atherosclerotic plaques, which is in agreement with the results of the morphometric test. It was found that low values of $\Delta n$ and IDF indicate the presence of a small amount of highly organized collagen in the fibrous cap of an unstable plaque which can be a sign of its tendency to rupture. We believe that these changes are connected with the prevalence of disorganized fibers in course of the inflammatory process in the fibrous cap of 
an unstable plaque and with the presence of clusters of foam cells and inflammatory cells between them.

The developed approach to quantitative evaluation of polarization properties of atherosclerotic plaques at different development stages can provide a basis for performing automated independent diagnostics of unstable plaques by CP OCT technique.

Acknowledgement. Numerical image processing was supported by the grant of the Russian Foundation for Basic Research No.15-32-20250. The medical and biological part of this work as well as work on the analysis of the efficiency of algorithms for image processing and selection of optimal quantitative characteristics were supported by the Russian Scientific Foundation, agreement No.14-15-00538 (EVG, MYuK, LBT, SSK, NDG).

Conflicts of Interest. The authors have no conflict of interests.

\section{References}

1. Brezinski M.E., Tearney G.J., Weissman N.J., Boppart S.A., Bouma B.E., Hee M.R., Weyman A.E., Swanson E.A., Southern J.F., Fujimoto J.G. Assessing atherosclerotic plaque morphology: comparison of optical coherence tomography and high frequency intravascular ultrasound. Heart 1997; 77(5): 397-404, http://dx.doi. org/10.1136/hrt.77.5.397.

2. Fujimoto J.G., Boppart S.A., Tearney G.J., Bouma B.E., Pitris C., Brezinski M.E. High resolution in vivo intra-arterial imaging with optical coherence tomography. Heart 1999; 82(2): 128-133, http://dx.doi.org/10.1136/hrt.82.2.128.

3. Yabushita H., Bouma B.E., Houser S.L., Aretz H.T., Jang I.K., Schlendorf K.H., Kauffman C.R., Shishkov M., Kang D.H., Halpern E.F., Tearney G.J. Characterization of human atherosclerosis by optical coherence tomography. Circulation 2002; 106(13): 1640-1645, http://dx.doi.org/10.1161/01. CIR.0000029927.92825.F6.

4. Jang I.K., Bouma B.E., Kang D.H., Park S.J., Park S.W., Seung K.B., Choi K.B., Shishkov M., Schlendorf K., Pomerantsev E., Houser S.L., Aretz H.T., Tearney G.J. Visualization of coronary atherosclerotic plaques in patients using optical coherence tomography: comparison with intravascular ultrasound. J Am Coll Cardiol 2002; 39(4): 604609, http://dx.doi.org/10.1016/s0735-1097(01)01799-5.

5. Stamper D., Weissman N.J., Brezinski M. Plaque characterization with optical coherence tomography. J Am Coll Cardiol 2006; 47(8): C69-C79, http://dx.doi.org/10.1016/ j.jacc.2005.10.067.

6. Brezinski M.E. Optical coherence tomography for identifying of unstable coronary plaque. Int J Cardiol 2006; 107(2): 159-170, http://dx.doi.org/10.1016/j.jicard.2005.07.066.

7. Tearney G.J., Yabushita H., Houser S.L., Aretz H.T., Jang I.K., Schlendorf K.H., Kauffman C.R., Shishkov M., Halpern E.F., Bouma B.E. Quantification of macrophage content in atherosclerotic plaques by optical coherence tomography. Circulation 2003; 107(1): 113-119, http://dx.doi.org/10.1161/01. cir.0000044384.41037.43.

8. Tearney G.J., Jang I.K., Bouma B.E. Optical coherence tomography for imaging the vulnerable plaque. J Biomed Opt 2006; 11(2): 021002, http://dx.doi.org/10.1117/1.2192697.
9. Kubo T., Xu C., Wang Z., Ditzhuijzen N.S., Bezerra H.G. Plaque and thrombus evaluation by optical coherence tomography. Int J Cardiovasc Imaging 2011; 27(2): 289-298, http://dx.doi.org/10.1007/s10554-010-9790-1.

10. Flueraru C., Popescu D.P., Mao Y., Chang S., Sowa M.G., Vitkin A. Improved arterial tissue differentiation by spectroscopic optical coherence tomography. Sovremennye tehnologii $v$ medicine 2015; 7(1): 13-20, http://dx.doi.org/10.17691/ stm2015.7.1.02.

11. Nadkami S.K., Pierce M.C., Park B.H., de Boer J.F., Whittaker P., Bouma B.E., Bressner J.E., Halpern E., Houser S.L., Tearney G.J. Measurement of collagen and smooth muscle cell content in atherosclerotic plaques using polarization-sensitive optical coherence tomography. J Am Coll Cardiol 2007; 49(13): 1474-1481, http://dx.doi.org/10.1016/j.jacc.2006.11.040.

12. de Boer J.F., Milner T.E., van Gemert M.J.C., Nelson J.S. Two dimensional birefringence imaging in biological tissue by polarization-sensitive optical coherence tomography. Opt Lett 1997; 22(12): 934-936, http://dx.doi.org/10.1364/ol.22.000934.

13. Lim Y., Yamanari M., Fukuda S., Kaji Y., Kiuchi T., Miura M., Oshika T., Yasuno Y. Birefringence measurement of cornea and anterior segment by office-based polarizationsensitive optical coherence tomography. Biomed Opt Express 2011; 2(8): 2392-2402, http://dx.doi.org/10.1364/boe.2.002392.

14. Liu B., Harman M., Giattina S. Characterizing of tissue microstructure with single-detector polarization-sensitive optical coherence tomography. Appl Opt 2006; 45(18): 4464-4479, http://dx.doi.org/10.1364/ao.45.004464.

15. Giattina S.D., Courtney B.K., Herz P.R., Harman M., Shortkroff S., Stamper D.L., Liu B., Fujimoto J.G., Brezinski M.E. Assessment of coronary plaque collagen with polarization sensitive optical coherence tomography (PS-OCT). Int J Cardiol 2006; 107(3): 400-409, http://dx.doi.org/10.1016/ j.ijcard.2005.11.036.

16. Nadkarni S., Pierce M., Park H., deBoer J., Houser S., Bressner J., Bouma B., Tearney G. Polarization-sensitive optical coherence tomography for the analysis of collagen content in atherosclerotic plaques. Circulation 2005; 112(17): U679-U679.

17. Kuo W.-C., Chou N.K., Chou C., Lai C.M., Huang H.J., Wang S.S., Shyu J.J. Polarization-sensitive optical coherence tomography for imaging human atherosclerosis. Appl Opt 2007; 46(13): 2520-2527, http://dx.doi.org/10.1364/ao.46.002520.

18. Kuo W.-C., Hsiung M.-W., Shyu J.-J., Chou N.-K., Yang P.-N. Assessment of arterial characteristics in human atherosclerosis by extracting optical properties from polarizationsensitive optical coherence tomography. Opt Express 2008; 16(11): 8117-8125, http://dx.doi.org/10.1364/oe.16.008117.

19. Gelikonov V.M., Gelikonov G.V. New approach to crosspolarized optical coherence tomography based on orthogonal arbitrarily polarized modes. Laser Physics Letters 2006; 3(9): 445-451, http://dx.doi.org/10.1002/lapl.200610030.

20. de Boer J.F., Srinivas S.M., Nelson J.S., Milner T.E., Ducros M.G. Polarization-sensitive optical coherence tomography. In: Handbook of optical coherence tomography. Bouma B.E., Tearney G.J. (editors). CRC Press 2001; p. 237274, http://dx.doi.org/10.1201/b14024-10.

21. Kuranov R.V., Sapozhnikova V.V., Turchin I.V., Zagainova E.V., Gelikonov V.M., Kamensky V.A., Snopova L.B., Prodanetz N.N. Complementary use of cross-polarization and standard OCT for differential diagnosis of pathological tissues. Opt Express 2002; 10(15): 707-713, http://dx.doi.org/10.1364/ OE.10.000707. 
22. Gubarkova E.V., Dudenkova V.V., Feldchtein F.I., Timofeeva L.B., Kiseleva E.B., Kuznetsov S.S., Shakhov B.E., Moiseev A.A., Gelikonov G.V., Vitkin A., Gladkova N.D. Multimodal optical imaging characterization of atherosclerotic plaques. J Biophotonics 2015; accepted for publication 04.11.15.

23. Kiseleva E., Kirillin M., Feldchtein F., Vitkin A., Sergeeva E., Zagaynova E., Streltzova O., Shakhov B., Gubarkova E., Gladkova N. Differential diagnosis of human bladder mucosa pathologies in vivo with cross-polarization optical coherence tomography. Biomed Opt Express 2015; 6(4): 1464-1476, http://dx.doi.org/10.1364/BOE.6.001464.

24. Gladkova N., Kiseleva E., Robakidze N., Balalaeva I., Karabut M., Gubarkova E., Feldchtein F. Evaluation of oral mucosa collagen condition with cross-polarization optical coherence tomography. J Biophotonics 2013; 6(4): 321-329, http://dx.doi.org/10.1002/jbio.201200059.

25. Gelikonov V., Gelikonov G., Shilyagin P. Optimization of Fizeau-based optical coherence tomography with a reference Michelson interferometer. Bulletin of the Russian Academy of Sciences: Physics 2008; 72(1): 93-97.

26. Gelikonov V.M., Gelikonov G.V., Shilyagin P.A. Linearwavenumber spectrometer for high-speed spectral-domain optical coherence tomography. Opt Spectrosc 2009; 106(3): 459-465, http://dx.doi.org/10.1134/s0030400x09030242.

27. Moiseev A.A., Gelikonov G.V., Terpelov D.A., Shilyagin P.A., Gelikonov V.M. Noniterative method of reconstruction optical coherence tomography images with improved lateral resolution in semitransparent media. Laser Physics Letters 2013; 10(12): 125601, http://dx.doi. org/10.1088/1612-2011/10/12/125601.

28. Stary H.C., Chandler A.B., Dinsmore R.E., Fuster V., Glagov S., Insull W.J., Rosenfeld M.E., Schwartz C.J., Wagner W.D., Wissler R.W. A definition of advanced types of atherosclerotic lesions and a histological classification of atherosclerosis: a report from the Committee on Vascular Lesions of the Council on Arteriosclerosis, American Heart Association. Arterioscler Thromb Vasc Biol 1995; 15(9): 15121531, http://dx.doi.org/10.1161/01.atv.15.9.1512.

29. Virmani R., Kolodgie P.D., Burke A.P., Farb A., Schwartz S.M. Lessons from sudden coronary death: a comprehensive morphological classification scheme for atherosclerotic lesions. Arterioscler Thromb Vasc Biol 2000; 20(5): 1262-1275, http://dx.doi.org/10.1161/01.atv.20.5.1262.

30. Ragino Y.I., Volkov A.M., Cherniavsky A.M. Stages of development of atherosclerotic hearth and types of unstable plaques - pathophysiological and histological characteristics. Rossiyskiy kardiologicheskiy zhurnal 2013; 5(103): 88-95.

31. Kiseleva E.B., Gladkova N.D., Sergeeva E.A., Kirillin M. Yu., Gubarkova E.B., Karabut M.M., Balalaeva I.V., Strel'tsova O.S., Robakidze N.S., Maslennikova A.V., Kochueva M.V. Sposob otsenki funktsional'nogo sostoyaniya kollagensoderzhashchikh tkaney. Zayavka na izobretenie No.2013135571 ot 29.07.2013 [Method for evaluating the functional state of collagen tissue. The application for the invention No.2013135571 from 29.07.2013].

32. Gubarkova E.V., Kirillin M.Yu., Sergeeva E.A., Kiseleva E.B., Snopova L.B., Prodanets N.N., Sharabrin E.G., Shakhov E.B., Nemirova S.V., Gladkova N.D. Cross-polarization optical coherence tomography in evaluation of atherosclerotic plaque structure. Sovremennye tehnologii v medicine 2013; 5(4): 45-55.

33. Kuo W.-C. Polarization-sensitive optical coherence tomography in cardiology. In: Advances in lasers and electro optics. InTech; 2010, http://dx.doi.org/10.5772/8660.

34. Tearney G.J., Regar E., Akasaka T., Adriaenssens T., Barlis P., Bezerra H.G., Bouma B., Bruining N., Cho J.-m., Chowdhary S., Costa M.A., de Silva R., Dijkstra J., Di Mario C., Dudeck D., Falk E., Feldman M.D., Fitzgerald P., Garcia H., Gonzalo N., Granada J.F., Guagliumi G., Holm N.R., Honda Y., Ikeno F., Kawasaki M., Kochman J., Koltowski L., Kubo T., Kume T., Kyono H., Lam C.C.S., Lamouche G., Lee D.P., Leon M.B., Maehara A., Manfrini O., Mintz G.S., Mizuno K., Morel M.-a., Nadkarni S., Okura H., Otake H., Pietrasik A., Prati F., Räber L., Radu M.D., Rieber J., Riga M., Rollins A., Rosenberg M., Sirbu V., Serruys P.W.J.C., Shimada K., Shinke T., Shite J., Siegel E., Sonada S., Suter M., Takarada S., Tanaka A., Terashima M., Troels T., Uemura S., Ughi G.J., van Beusekom H.M.M., van der Steen A.F.W., van Es G.-A., van Soest G., Virmani R., Waxman S., Weissman N.J., Weisz G. Consensus standards for acquisition, measurement, and reporting of intravascular optical coherence tomography studies. A report from the International Working Group for Intravascular Optical Coherence Tomography Standardization and Validation. J Am Coll Cardiol 2012; 59(12): 1058-1072, http://dx.doi. org/10.1016/j.jacc.2011.09.079.

35. Virmani R., Burke A.P., Farb A., Kolodgie P.D. Pathology of unstable plaque. Prog Cardiovasc Dis 2002; 44(5): 349-356, http://dx.doi.org/10.1053/pcad.2002.122475. 